\title{
PSMA-Positive Tumor
}

National Cancer Institute

\section{Source}

National Cancer Institute. PSMA-Positive Tumor. NCI Thesaurus. Code C148035.

An indication that prostate-specific membrane antigen (PSMA) expression has been detected in a tumor sample. 